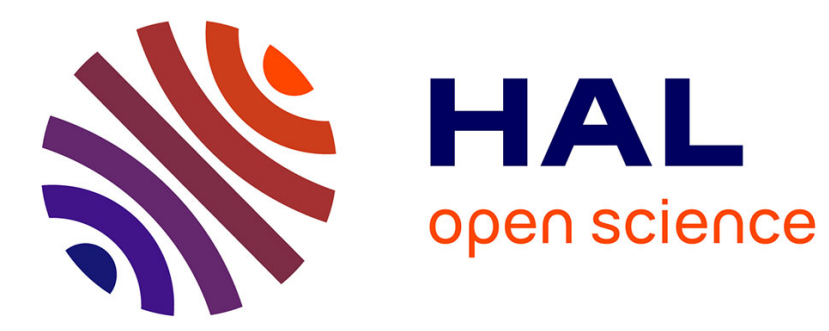

\title{
La Seconde commission de sauvegarde des droits et libertés individuels
}

\author{
Raphaëlle Branche
}

\section{To cite this version:}

Raphaëlle Branche. La Seconde commission de sauvegarde des droits et libertés individuels. La justice en Algérie 1830-1962, 2002, Paris, France. p.237-246. halshs-00541824

\section{HAL Id: halshs-00541824 \\ https://shs.hal.science/halshs-00541824}

Submitted on 1 Dec 2010

HAL is a multi-disciplinary open access archive for the deposit and dissemination of scientific research documents, whether they are published or not. The documents may come from teaching and research institutions in France or abroad, or from public or private research centers.
L'archive ouverte pluridisciplinaire HAL, est destinée au dépôt et à la diffusion de documents scientifiques de niveau recherche, publiés ou non, émanant des établissements d'enseignement et de recherche français ou étrangers, des laboratoires publics ou privés. 


\section{La Seconde commission de sauvegarde des droits et libertés individuels}

in AFHJ, La justice en Algérie 1830-1962, Paris, La Documentation Française, 2005, 366 p., p.237-246.

La commission de sauvegarde des droits et libertés individuels, reformée à l'été 1958, entre en scène dans une guerre d'Algérie déjà vieille de près de quatre ans et habituée à des accommodements certains avec le droit ${ }^{1}$. Une législation d'exception a été mise en place dès le début de la guerre et la justice a dû s'acclimater à cette situation particulière, que ce soit dans les principes ou dans les faits. La commission de sauvegarde est chargée de s'assurer de la préservation de certains droits en Algérie et en métropole, droits qui sont - l'opinion publique comme les autorités politiques le savent bien - bafoués régulièrement par les forces de l'ordre engagées dans la lutte contre le nationalisme algérien.

Face à ces violations, la commission de sauvegarde ne fonctionne pas comme une justice de guerre (pour reprendre le titre de la demi-journée). Plus largement, il semble bien qu'il soit très difficile politiquement de faire traduire les auteurs de ces actes devant une justice quelle qu'elle soit: les forces de l'ordre, et notamment l'armée, imposent leurs méthodes en Algérie et les pouvoirs publics n'ont pas les moyens - en tout cas en 1958 - de les contrer.

En revanche, la commission de sauvegarde constitue bien une forme originale d'intervention $\mathrm{du}$ droit dans la guerre: elle vient rappeler certains principes fondamentaux de la République française, en l'occurrence, le respect nécessaire des droits et libertés individuels de chacun, a priori. Faut-il alors comprendre son existence comme un désir politique de maintenir présents ces principes tels des impératifs catégoriques proposés aux forces de l'ordre? Faut-il y voir un souci moral d'assurer, au sein de la guerre et des violations du droit qui l'accompagnent, la survie de ce qui semble pourtant compromis par les actes mêmes des forces de l'ordre? Ces dimensions existent assurément. Elles ne doivent pas gommer le fait que la commission de sauvegarde a pu avoir un rôle actif dans la modification de la justice pendant la guerre dans un sens plus répressif : on peut d'ailleurs se demander quels liens il a existé entre ces actions-là et la mission de la commission. Au-delà de son titre officiel, on peut se demander ce que cette commission était chargée de sauvegarder et ce qu'elle a réellement sauvegardé2 ?

Quand elle a été créée par le gouvernement Mollet, la commission avait indubitablement pour but de sauvegarder du scandale la France et son gouvernement. Constituée en effet juste après l'émergence dans la presse métropolitaine et étrangère, de dénonciations récurrentes des méthodes employées par l'armée française en Algérie et notamment à Alger, la commission de sauvegarde devait s'assurer de la véracité de ces accusations, faire la lumière sur les calomnies

\footnotetext{
${ }^{1}$ Le fonctionnement et les missions de la commission sont fixés par l'ordonnance n`58-739 du 20 août 1958.

${ }^{2}$ L'étude de son fonctionnement permet d'éclairer les relations nouées pendant la deuxième moitié de la guerre d'Algérie entre droit, justice et guerre. Elle est aujourd'hui possible grâce à l'ouverture de ses archives conservées aux Archives Nationales (AN).
} 
éventuelles mais aussi sur les violations réelles perpétrées en Algérie. Cette première commission a eu une période d'activité de quelques mois - ce qui s'explique par le contexte opportuniste de sa création ${ }^{3}$.

Il en est apparemment tout autrement de la commission que le général De Gaulle souhaite relancer dès son retour aux affaires. Soucieux de marquer l'intérêt de l'Etat pour la question des droits et libertés individuels, le Général a nommé à la tête de la nouvelle commission un homme important: Maurice Patin, président de la chambre criminelle de la cour de cassation ${ }^{4}$. Les membres de la commission sont choisis pour leur notoriété dans les milieux judiciaires, militaires, médicaux ou encore universitaires. Certains sont conseillers $\mathrm{d}^{\prime}$ Etat $^{5}$. Ils sont tous bénévoles et $\mathrm{d}$ 'ailleurs bien souvent très accaparés par d'autres taches que celles de la commission. Manifestement un certain souci d'équilibre politique a également prévalu lors de la composition de la commission.

Les autorités veulent toujours éviter les scandales qui pourraient éclabousser l'action de la France mais la commission est, cette fois, chargée d'un travail au long cours. À la tête de l'Etat, certains sont fermement décidés à accélérer la « recherche et la poursuite des exactions commises par des militaires ou des agents de la force publique $»^{6}$. Le général De Gaulle est de ceux-là : il aurait affirmé à Maurice Patin vouloir « donner à l'opinion des apaisements indiscutables et définitifs $»^{7}$. C'est ainsi qu'on peut comprendre sa volonté exprimée plusieurs fois $\mathrm{d}^{\prime}$ une plus grande visibilité de la commission notamment en Algérie où il a été envisagé d'installer un ou plusieurs représentants permanents. Le souci d'éviter les scandales a aussi pu toucher des gens comme le général Massu qui, dès la première visite de la commission en Algérie, début septembre 1958, saisit son président du statut civil "d'environ 3000 femmes musulmanes dont les maris ont disparu au cours des événements de février 1957 ». Le sort de ces femmes, victimes de l'action des troupes du général Massu lui-même, avaient en effet touché Madame Massu qui s'était inquiétée de leur statut, puisqu'elles n'étaient pas officiellement veuves.

Les missions de la commission sont resserrées sur la question de la violation des droits - l'évaluation du caractère calomnieux de certaines accusations disparaît et son cadre $\mathrm{d}$ 'action élargi à la métropole. Son fonctionnement demeure à peu près le même: des enquêtes directes des membres de la commission, d'une part, et des demandes d'enquête à la gendarmerie. Les rapports de la commission ne sont pas publics. Ils sont destinés au président du Conseil puis, sous la Ve République, au Premier ministre. C'est le secrétariat général aux affaires algériennes qui est chargé de suivre leur exécution soit en saisissant le délégué général en Algérie, soit le ministre des Armées. Le général De Gaulle en est aussi informé mais les ministres,

\footnotetext{
${ }^{3}$ Voir R.Branche, «La Commission de sauvegarde des droits et libertés individuels pendant la guerre d'Algérie. Chronique d'un échec annoncé? », Vingtième Siècle. Revue d'histoire, n 62 , avril-juin 1999, p. 14-29.

${ }^{4}$ Sur Maurice Patin, voir notamment Thénault et Branche, « Debré..... »

${ }^{5}$ Ils sont nommés par décret le 6 septembre 1958. Il s'agit, outre Maurice Patin, de messieurs Damour, Daure, François-Poncet, Haag,

Ledoux, Moliérac, Oudinot, Richet, de Segogne, de Vernejoul et Zeller. Certains démissionnent avant la fin de la commission. D'autres sont nommés au cours de la guerre.

${ }^{6}$ Lettre de Chaban-Delmas, ministre de la Défense Nationale et des forces armées au général Salan le 30 janvier 1958 , F60 3147 (AN).

${ }^{7}$ Lettre de Patin à Brouillet, le 4 août 1958.
} 
eux, n'en reçoivent copie que quand ils sont directement concernés et engagés à agir de même pour le délégué général en Algérie8.

Rapidement la commission devient l'interlocuteur officiel en charge des questions des violations des droits et libertés individuels : les lettres d'Algériens se multiplient ; les avocats et les groupes militants la saisissent. C'est ainsi que le comité Audin est reçu plusieurs fois par le président Patin - le secrétaire général de la commission accompagnant ses membres chez le garde des Sceaux pour une audience fin $1958^{\circ}$. Un an plus tard, c'est même à la demande du garde des Sceaux et du général De Gaulle que Maurice Patin reçoit le comité Audin pour lui demander de « cesser toute publication intempestive dans la presse, ce qui risquerait de contrarier la marche normale de l'instruction en cours $»^{10}$.

La commission tente de donner suite à toutes les informations qui lui parviennent mais elle a le souci de ne pas se laisser dicter le choix des affaires sur lesquelles intervenir. Le président Patin y voit une affirmation de son indépendance, gage de sa crédibilité notamment auprès des Algériens. Il a en même temps le souci de ne pas gêner le gouvernement, et surtout le président de la République. Ainsi, lors de l'instruction de l'affaire Audin à Rennes - instruction qui avait lieu en métropole, la commission ayant souhaité que l'affaire soit transférée d'Algérie en France -, le président Patin refuse que le rapport du recteur Daure sur Audin, réalisé lors de la première commission de sauvegarde soit versé au dossier. Il autorise en revanche le recteur à se rendre à la convocation du juge d'instruction mais préfère que la commission ne soit pas engagée davantage dans cette affaire très médiatisée ${ }^{11}$.

Sur l'affaire Audin comme sur d'autres, la position de la commission est souvent ambiguë. Ses membres peuvent, dans le cadre de leurs enquêtes, se retrouver à mener ce qui ressemble beaucoup à une information judiciaire. Chez les plaignants, cette ambiguïté peut faire naître des espoirs ; des obstructions du côté des forces de l'ordre peuvent aussi s'expliquer par là. En fait, quand elle est persuadée du bien-fondé d'une plainte, la commission ne peut instruire quoique ce soit et se contente de rédiger un rapport ou/et de transmettre ses conclusions aux juridictions compétentes. Ainsi, elle participe à l'action de la justice dans la guerre - sans pouvoir agir directement. Dans le cas d'affaires très médiatisées comme la disparition de Maurice Audin ou les sévices subis par Djamila Boupacha, Maurice Patin s'enquière régulièrement de l'avancée des dossiers et tente de les suivre de près ${ }^{12}$. Il ne peut faire plus.

En revanche, la commission de sauvegarde est associée, en particulier par l'intermédiaire de son président, aux mutations que connaît la justice pendant cette deuxième moitié de la guerre. Maurice Patin est chargé de rapports sur ces questions ; il donne régulièrement son avis aux ministres concernés. Les observations qu'il peut faire au sein de la commission de sauvegarde le persuadent qu'il est

\footnotetext{
${ }^{8}$ Il s'en plaint amèrement dans une lettre au Premier ministre du 26 août 1959, cab 14/84 (CAOM)

${ }^{9}$ Procès-verbal de la réunion de la commission du 9 janvier $1959, \mathrm{~F}^{60} 3134$.

${ }^{10}$ Procès-verbal de la séance du 19 décembre $1959, \mathrm{~F}^{60} 3134$.

${ }^{11}$ Procès-verbal de la séance du 17 juillet $1959, \mathrm{~F}^{60} 3134$.

${ }^{12}$ Dossier sur l'affaire Audin $\mathrm{F}^{60} 3165$.
} 
nécessaire de "doter l'armée d'Algérie d'une justice efficace et de lui assurer la collaboration de la Magistrature ${ }^{13}$. Il qualifie lui-même ce souci "d'action préventive » : il s'agit d'éviter que les militaires ne recourent à une justice expéditive, qualifiée de parallèle, sous prétexte que la justice ordinaire n'est pas assez rapide et efficace.

Maurice Patin estime qu'une réforme de la justice militaire en Algérie est indispensable, dans un premier temps par la multiplication des tribunaux de zone en substitution des trois uniques tribunaux militaires existant au niveau des trois corps d'armée, puis, dans un second temps, par la création d'un corps de procureurs militaires travaillant au plus près des opérations militaires, en collaboration intime avec les responsables militaires des secteurs ${ }^{14}$. Il s'agit - comme il l'expose en réunion du comité des affaires algériennes - $d^{\prime}$ " améliorer le fonctionnement de la justice et [d']assurer le respect des droits de l'homme ». Ces deux buts ne pouvant être atteints - le président Patin en acquiert rapidement la certitude - qu'avec le «concours des chefs de l'administration, de la police ou de l'armée $»^{15}$.

De fait, estime un des membres de la commission, «l'autorité, le prestige, le grand caractère, le crédit» dont jouit Maurice Patin «auprès de certains chefs militaires [... donnent] à son action personnelle une puissance de persuasion à laquelle ne pourrait prétendre l'anonymat agressif d'une censure collégiale ${ }^{16}$. Le président Patin use en tout cas beaucoup de son influence pour convaincre les autorités militaires du bien-fondé des réformes qu'il propose et de la nécessité de respecter les droits que sa commission est censée sauvegarder. Il joue constamment de ces deux fonctions - auxquelles il faut ajouter son rôle de président de la chambre criminelle de la cour de cassation ${ }^{17}$. Lors de la composition de la commission, il demande d'ailleurs à ce que soient nommés avec lui deux conseillers de cette chambre en qui il aurait une grande confiance. Il y voit un moyen de «mieux marquer que la sauvegarde des libertés est placée, pour une large part, entre les mains de magistrats inamovibles ». Il s'agit clairement d'un souci d'indépendance et de liberté, qui se manifeste aussi par son désir d'avoir une liberté absolue d'intervention dans les lieux de détention ou d'interrogatoire et d'avoir le pouvoir d'exiger des toutes les autorités des explications ou des documents sur ce qui lui semblera bon ${ }^{18}$.

On peut y voir aussi la marque du haut magistrat pénétré de l'importance extrême de sa mission. Au cours des réunions de la commission, il n'a de cesse de rappeler à ses membres leur rôle unique : affichant un gaullisme absolu, il se veut avant tout au service du Général et quasiment son représentant sur le terrain politiquement sensible des droits de l'homme. Les membres de la commission ne

\footnotetext{
${ }^{13}$ Lettre de Maurice Patin au général Cantarel, commandant le CAO, 17 janvier 1962, $1 \mathrm{H} 1240 / 9$ (SHAT).

${ }^{14}$ Voir Sylvie Thénault, Une drôle de justice....

${ }^{15}$ Compte-rendu de la séance du 5 octobre 1960 du comité des affaires algériennes, $\mathrm{F}^{60} 3146$

${ }^{16}$ Rapport de Fernand Grévisse, 25 septembre 1959, $\mathrm{F}^{60} 3144$.

${ }^{17}$ Le rapport qui remet au Premier ministre sur l'application du décret du 12 février 1960 est tout à fait exemplaire sur ce point. Il y mêle des considérations inspirées de son rôle de président de la commission de sauvegarde et d'autres liées à son rang dans la magistrature, le tout justifié par son souci d'éviter à la France un coup d'Etat... Rapport sur sa mission du 6 au 10 avril $1960, \mathrm{~F}^{60} 3147$.

${ }^{18}$ Lettre de Maurice Patin à René Brouillet, 4 août 1958.
} 
peuvent qu'acquiescer à cette vision des choses ou se démettre. Présentant la commission à quatre nouveaux membres en décembre 1961, Maurice Patin n'hésite d'ailleurs pas à exposer les choses ainsi : «La commission se trouve maintenant face à de nouvelles tâches. Le gouvernement lui-même se trouve aux prises avec de nouvelles difficultés. Il se trouve que notre tâche est telle qu'elle doit empêcher le gouvernement et les autorités de sortir de la légalité »- tout en ajoutant que «c'est tout cela qui a pu motiver le départ de certains ${ }^{19}$ !

Sur le terrain, la commission est épaulée par des collaborateurs extérieurs importants. En Algérie, elle travaille souvent avec le commandant Arnoux, chargé du bureau de la justice militaire auprès du commandant du Corps d'Armée d'Alger. Maurice Patin estime avoir en lui un interlocuteur efficace et de bonne foi, ayant en particulier plaidé avec succès la cause de la justice auprès du général Massu' ${ }^{20}$. Son influence est jugée essentielle par le président Patin qui a bien compris que «la sauvegarde des droits et libertés [était] avant tout en Algérie un problème de commandement $»^{21}$. En France, elle collabore très régulièrement avec Monsieur Viatte, responsable de la commission d'inspection des centres d'internement en métropole. Il devient d'ailleurs membre de la commission de sauvegarde en novembre 1961. Avec le pendant de cette commission en Algérie, la commission dirigée par les généraux Boyer-Vidal puis Durand, les rapports semblent beaucoup moins réguliers ${ }^{22}$. Ces collaborations sont extrêmement importantes pour assurer à la commission un suivi sur le terrain. Autrement elle ne serait condamnée qu'à des interventions ponctuelles.

Aux yeux du président Patin cependant, ni les réformes successives de la justice militaire, ni les actions de sa commission ne peuvent suffire à sauvegarder les droits et libertés individuels en Algérie et à éliminer toute "justice parallèle ». Les membres de la commission ont entendu les explications de certains militaires; ils tentent de faire la part des impératifs opérationnels et des violations intolérables des droits.

Un mot exprime le champ d'intervention à l'horizon de l'action de la commission de sauvegarde : la morale. C'est elle que Maurice Patin tente de faire prévaloir au sein des forces de l'ordre ${ }^{23}$. La commission renouvelle pour cela régulièrement son souci non seulement que la justice règne en Algérie, mais aussi que des consignes «de retenue » soient données «conviant impérativement et sous peine de sanctions graves à n'agir qu'avec circonspection et dans le cadre des formes légales $»^{24}$. Interrogé près de quarante ans plus tard, un membre de la commission

\footnotetext{
${ }^{19} \mathrm{PV}$ de la séance du 16 décembre $1961, \mathrm{~F}^{60} 3134$.

${ }^{20}$ Correspondance entre Maurice Patin et le commandant Arnoux, $\mathrm{F}^{60} 3144$.

${ }^{21}$ Rapport de Maurice Patin au Premier ministre sur sa mission en Algérie du 29 mai au 3 juin 1959, $\mathrm{F}^{60} 3147$.

${ }^{22}$ Sur la commission d'inspection des centres de détention administrative, voir R.Branche, « « Comment rétablir de la norme en temps d'exception. L'IGCI/CICDA pendant la guerre d'Algérie » in Laurent Feller (dir.), Contrôler les agents du pouvoir, Limoges, PULIM, 2004, p.299-310.

${ }^{23}$ Citons, par exemple, son rapport au Premier ministre sur sa mission en Algérie du 29 janvier au 2 février 1959 : « Nous nous sommes efforcés, bien que la tâche soit difficile, de nous éclairer aussi complètement que possible, sur l'action des diverses polices existant en Algérie, polices relativement nombreuses, puisqu'il s'ajoute, à la gendarmerie et à la police d'Etat, la police militaire, effectuée dans chaque unité par les OR, ainsi que par les DOP. Nous n'avons malheureusement pas pu acquérir la certitude qu'il n'était pas recouru à des moyens d'investigation que la loi et la morale n'admettent pas, tels que l'emploi de l'eau ou de l'électricité, et que beaucoup considèrent comme légitime, par le motif que la défense sociale devrait primer la sauvegarde des individuels ». F60 3147 (c'est nous qui soulignons). ${ }^{24}$ Rapport de M. de Ségogne sur la mission qu'il a menée en Algérie avec M. Moliérac du 8 au 13 juillet $1959, \mathrm{~F}^{60} 3148$.
} 
résume leur but en une formule : "Humaniser la guerre d'Algérie », soulignant en même temps «l'antagonisme évident entre cette mission et la guerre elle-même » ${ }^{25}$ qui rendait leur action très difficile ${ }^{26}$.

De nombreux membres de la commission sont désespérés par le peu de résultats qu'ils obtiennent. Le président Patin a, certes, pu observé une évolution de la justice militaire dans le sens souhaité - pour ôter aux militaires le prétexte d'une justice peu efficace-, peut-il pour autant affirmer que la «justice parallèle»a disparu? Il tente de le faire, chiffres d'exécutions sommaires à l'appui, mais la réalité est plus complexe ${ }^{27}$. Quant à la mission de la commission de sauvegarde stricto sensu, l'analyse de son bilan conduit à se demander ce qu'elle a finalement sauvegardé.

À lire les plaintes exprimées régulièrement par les membres de la commission au cours de leurs réunions, il n'y aucune doute : leur insatisfaction ne cesse pas et les nouveaux membres viennent rapidement rejoindre les autres sur ce terrain. Cette insatisfaction tient tout d'abord au fonctionnement de la commission proprement dit. Celle-ci repose avant tout sur Maurice Patin qui, accompagné de Louis Damour, se rend très régulièrement en Algérie, et qui fait la liaison entre le gouvernement et les membres de la commission. Sans lui, la commission ne peut pas fonctionner - la meilleure preuve en est qu'elle suspend de fait ses activités entre fin mars et fin octobre 1961 : son président ayant été appelé à d'autres fonctions ${ }^{28}$. Les membres de la commission n'ont quasiment aucun contact avec les autorités politiques qui les ont nommés : ils sont frustrés de cette absence qu'ils assimilent à un silence et finalement à une absence de reconnaissance.

De fait, aucune publicité n'est donnée à leurs rapports et bien souvent aucun accusé de réception ne vient même signaler que les ministres concernés les ont lus ${ }^{29}$ ! Lors de la réorganisation de la justice militaire, au printemps 1960, la commission de sauvegarde se voit bien invitée à « poursuivre sa mission $»^{30}$, mais aucune distinction honorifique, par exemple, ne témoigne officiellement de la valeur qu'on lui accorde. Si Maurice Patin est devenu grand officier de la légion d'honneur, aucun autre membre ou collaborateur de la commission n'est distingué.

Même si Michel Debré présente «la discrétion observée au sujet des travaux de la commission » comme ayant été motivée non seulement par « le désir de ne pas livrer à une regrettable publicité les faits révélés par [ses] investigations mais encore [par le] souci de ne pas [la] gêner dans son action $»^{31}$, certains de ses membres s'estiment injustement privés de reconnaissance officielle ${ }^{32}$. En désaccord parfois

\footnotetext{
${ }^{25}$ Entretien de Fernand Grévisse avec l'auteur, 7 février 1997.

${ }^{26}$ L'expression se trouve aussi dans la bouche de Maurice Patin. À propos de l'internement administratif, il expose ainsi sa position aux membres de la commission : «Cette mesure existe légalement. Nous en devons pas gêner le gouvernement »; « le principe de l'internement administratif est légal. Nous ne pouvons rien contre cela. Ce qui nous incombe c'est d'humaniser cette mesure ». Sa réponse est tout à fait typique des limites a priori fixées à l'action de la commission. Procès-verbal de la séance du 16 décembre $1961, \mathrm{~F}^{60} 3134$.

${ }^{27}$ Pour une discussion sur ce point, voir Thénault, Branche, déjà cité.

${ }^{28}$ Voir la communication de Sylvie Thénault dans ce même volume.

${ }^{29}$ Une note de Michel Debré à Pierre Racine confirme que cette impression peut correspondre à une réalité : j’ai le sentiment, écrit le Premier ministre, «que les conclusions de la commission sont envoyées dans les ministères où elles ont tendance à être enterrées ». Note du 4 janvier 1960, 2DE 20 (CHEVS). Fin 1961, le Premier ministre constate exactement la même situation (note à M. Morris du 11 octobre 1961).

${ }^{30}$ Relevé des décisions du comité des affaires algériennes, séance du 10 mai 1960, F 3146.

${ }^{31}$ Lettre de Michel Debré à Maurice Patin, le 3 mars 1960, $\mathrm{F}^{60} 3145$.

${ }^{32}$ Citons, à titre exceptionnel, la présence de Louis Joxe, invité par Maurice Patin a assisté à la fin de la séance de la commission du 18 novembre 1961. Le ministre des affaires algériennes y exprime son désaccord avec le ministre de l'intérieur à propos de l'immigration
} 
avec la politique menée par le gouvernement, ils sont en outre tenus au secret professionnel: quelques uns choisissent alors de démissionner ${ }^{33}$. D'autres se contentent de caresser l'idée sans franchir le pas. Maurice Patin, lui-même, abjure régulièrement ses collaborateurs de rester : l'inverse revenant, selon lui, à trahir le gouvernement et surtout le général De Gaulle. Le président de la commission n'est pourtant pas naïf : "Tout se passe comme si personne ne voulait convenir [de l'action bénéfique et indispensable de la commission], probablement parce que personne ne veut convenir des abus et des excès dont l'existence n'était que trop certaine et que la commission a combattus », écrit-il ainsi à Michel Debré34.

La commission de sauvegarde n'est pas dupe des camouflages utilisées par les forces de l'ordre pour maquiller les violations des droits qu'elles commettent. Elle décode les «fuyards abattus» en exécutions sommaires ${ }^{35}$. Elle sait qu'un procèsverbal de gendarmerie pour « évasion », « remise en liberté » ou «tentative de fuite » laisse « rarement de doute sur les conditions réelles de la disparition : mort sous la torture, liquidation physique du suspect ». Mais sa marge d'action est faible. Elle n'a pas les moyens d'enquêter sur tous ces cas ni de demander qu'on le fasse. Elle ne veut pas risquer d'être taxée $d^{\prime}$ " instruire le procès de l'Armée française » ${ }^{36}$.

Elle opte donc pour une solution intermédiaire: pour chaque disparition, informer les autorités concernées qu'elle a été saisie, l'espoir étant alors que ce signalement puisse fonctionner comme une protection pour la personne « disparue » si elle n'est que détenue au secret et non morte. La commission adopte le même système pour les individus arrêtés ou assignés à résidence dont on l'informe : là encore, le but est d'être une protection contre l'arbitraire.

Pour les victimes des forces de l'ordre, elle semble bien être apparue comme une sorte de recours. D'ailleurs, en juillet 1962, un membre de l'exécutif provisoire estime que la commission a «contribué beaucoup à éviter la rupture entre les musulmans algériens et la France ${ }^{37}$. Mais, dans les faits, la commission n'a été qu'une chambre d'enregistrement des violations des droits et libertés individuels par les forces de l'ordre.

Une vision optimiste a pu tenter de mettre en avant « des résultats sérieux bien que non spectaculaires $»^{38}$. Elle s'opposait à ceux qui parlaient de son « inefficacité d'ensemble » ${ }^{39}$. Mais les évolutions de la fin de la guerre rendent tout optimisme difficile à maintenir : "On a l'impression que la commission devient une couverture pour le gouvernement», note ainsi Maurice Patin, dépité. "Malgré les efforts faits, il a été impossible jusqu'ici d'émouvoir le gouvernement. [...Or,] il y a

\footnotetext{
algérienne en France et est interrogé par Maurice Patin sur les commissions Bertrand et Durand avec lesquelles la commission de sauvegarde avait l'habitude de travailler. Cette visite est l'unique fois où les membres de la commission peuvent dialoguer avec un ministre. Voir les PV des séances, $\mathrm{F}^{60} 3134$.

${ }^{33}$ À titre d'exemple, on peut citer le conseiller d'Etat Combaldieu qui démissionne en octobre 1961. A la séance de la commission qui avait précédé, en mars 1961, il s'était demandé à haute voix : «Comment se fait-il qu'aucun membre du gouvernement ne proteste contre la torture? ». Personne ne lui avait répondu. Messieurs Fernand Grévisse, Charles Richet, Roger de Ségogne et Henri Zeller ont aussi démissionné en 1961. M. Viatte fait de même en 1962.

${ }^{34}$ Lettre du 14 avril $1961, \mathrm{~F}^{60} 3144$.

${ }^{35}$ Sur ces camouflages, voir R.Branche, La torture et l'armée pendant la guerre d'Algérie, Gallimard, 2001 , 464 p., chapitre 3.

${ }^{36}$ Rapport de Fernand Grévisse, 25 septembre 1959, $\mathrm{F}^{60} 3144$.

${ }^{37}$ Propos de $\mathbf{M}^{\mathrm{e}}$ Saddor Kaddour rapportés par MM. Hersant et Meigné lors de la séance de la commission du 7 juillet 1962.

${ }^{38}$ Maurice Patin, séance du 4 mars 1959.

${ }^{39}$ Rapport de Fernand Grévisse déjà cité.
} 
une aggravation nouvelle : par suite de la lutte difficile entre le FLN et l'OAS, le gouvernement est amené à prendre des mesures exceptionnelles, à mettre en œuvre des moyens exorbitants. Il faut bien reconnaître que la législation de droit commun est insuffisante. Mais il en résulte que les libertés ne sont plus assurées. [...] Le gouvernement ferait bien de reconnaître de bonne foi qu'il est obligé de porter atteinte aux libertés individuelles $»^{40}$. A la fin de la guerre, ce constat amer est partagé par tous. André François-Poncet l'exprime ainsi: nous avons été des gêneurs, instrumentalisés par le gouvernement selon une chronologie politique qui ne laissait aucune liberté d'action réelle à la commission. Les violences d'octobre 1961 à Paris déclenchent un sentiment d'exaspération: "Devant une poussée d'opinion qui dénonce les brutalités, le gouvernement - pour avoir la paix - peut être tenté de faire apparaître à nouveau la commission. Puis on nous laissera de retomber dans l'oubli ${ }^{41}$.

Le sens ultime de la commission de sauvegarde ne serait-il pas à trouver dans la recherche d'un équilibre entre le gouvernement, les forces de l'ordre et l'opinion publique? Lors de sa création en avril 1957, la commission de sauvegarde avait très nettement pour vocation de limiter l'ampleur du scandale des violations des droits et des libertés individuels accomplies par les forces de l'ordre en Algérie. Avec l'arrivée du général De Gaulle au pouvoir, la réalité de ces violations avait été admise par le gouvernement (en la personne d'André Malraux), la fermeté des principes réaffirmée - tandis qu'en Algérie, l'armée recevait une grande liberté. Entre temps l'affaire algérienne s'était aussi internationalisée et l'opinion publique nationale avait montré son poids dans l'évolution politique du pays (par ses votes mais aussi ses mobilisations). La commission de sauvegarde nouvelle mouture était à l'image du nouveau contexte et à la taille des nouveaux enjeux.

Puisque ses pouvoirs semblaient importants, elle attira les plaintes visant les forces de l'ordre françaises et constitua un véritable canal de dérivation permanent vis-à-vis du cours normal de la justice. Mais sa capacité d'action était en réalité limitée et rares furent les affaires qui aboutirent judiciairement grâce à son soutien. En revanche la commission fut un véritable observatoire des violations au service du Premier ministre et du président de la République, un gisement de rapports et d'expertises pour modifier l'appareil judiciaire ou, plus simplement, pour rappeler les représentants des forces de l'ordre au respect de la légalité. Enfin, elle était, par son existence même, la preuve que la France continuait à être le pays des droits de l'homme. Cette image valait bien une commission de sauvegarde!

\footnotetext{
${ }^{40}$ Maurice Patin, séance du 28 octobre 1961.

${ }^{41}$ André François-Poncet, séance du 28 octobre 1961.
} 
